\title{
Second-order coplanar perturbation of a semi-infinite crack in an infinite body
}

\author{
Jean-Baptiste Leblond ${ }^{*}$, Sylvain Patinet ${ }^{2,3}$, Joël Frelat ${ }^{1}$, \\ Véronique Lazarus ${ }^{2}$ \\ ${ }^{1}$ UPMC Univ Paris 6 and CNRS, UMR 7190, Institut Jean Le Rond d'Alembert, F-75005 \\ Paris, France \\ ${ }^{2}$ UPMC Univ Paris 6, Univ Paris-Sud 11 and CNRS, UMR 7608, Lab FAST, F-91405 Orsay, \\ France \\ ${ }^{3}$ UPMC Univ Paris 6, Univ Paris 7 Diderot, ESPCI and CNRS, UMR 7636, Lab PMMH, \\ F-75005 Paris, France
}

\begin{abstract}
There has been considerable interest in recent years in theoretical formulae providing, for various crack configurations, the local variation of the stress intensity factors resulting from some small but otherwise arbitrary coplanar perturbation of the crack front. In this work, we establish the expression of the variation of the mode I stress intensity factor up to second order in the perturbation, in the specific case of a semi-infinite tensile crack embedded in some infinite body. The treatment is basically simple and uses earlier results of Rice (1989). Formulae are given in both the physical space and Fourier's space. They differ from earlier ones established by Adda-Bedia et al. (2006) for the same problem, using a more complex method of solution. Finite element computations performed for sinusoidal perturbations support the new formulae, rather than the older ones. As an application, it is finally shown that the mean value of the energy-release-rate along the front of a perturbed semi-infinite tensile crack is exactly the same, up to second order in the perturbation, as if the front were straight.
\end{abstract}

Keywords : Semi-infinite crack; coplanar perturbation; second order; stress intensity factor; fundamental kernel

\section{Introduction}

In the past 25 years, formulae providing the distribution of the stress intensity factors (SIF) for planar cracks with slightly perturbed fronts have received considerable attention. Such formulae have been established for a semi-infinite crack loaded in mode I (Rice, 1985) or arbitrarily (Gao and Rice, 1986); an internal circular crack loaded in mode I (Gao and Rice, 1987a) or arbitrarily (Gao, 1988); an external circular tensile crack (Gao

* Corresponding author. 
and Rice, 1987b); a tunnel-crack loaded in mode I (Leblond et al., 1996) or arbitrarily (Lazarus and Leblond, 2002a,b); a semi-infinite interface crack loaded arbitrarily (Lazarus and Leblond, 1998a,b; Piccolroaz et al., 2007); a pair of coplanar, parallel, identical tensile tunnel-cracks (Pindra et al., 2010); a pair of coplanar semi-infinite tensile cracks (Legrand and Leblond, 2010); and an emerging tensile crack lying on the mid-plane of a semi-infinite plate (Legrand et al., 2011).

The existence of these formulae has opened the way to theoretical studies of the evolution in time of the deformation of the front of cracks propagating in materials with heterogeneous fracture properties, in various situations: a tunnel-crack loaded in mode I (Favier et al., 2006) or arbitrarily (Pindra et al., 2009); a semi-infinite interface crack loaded arbitrarily (Pindra et al., 2008); and a pair of coplanar semi-infinite tensile cracks (Legrand and Leblond, 2010). From a more practical point of view, Rice (1985)'s formula for perturbation of a semi-infinite tensile crack has also been extensively used to assist the interpretation of experiments of separation of bonded plates; see the works of Schmittbuhl et al. (1995), Tanguy et al. (1998), Schmittbuhl and Vilotte (1999), Krishnamurthy et al. (2000), Roux et al. (2003), Schmittbuhl et al. (2003), Charles et al. (2004), Katzav and Adda-Bedia (2006), Bonamy et al. (2008), Laurson et al. (2010), Ponson and Bonamy (2010), and the reviews of Alava et al. (2006) and Lazarus (2011). Very recently, Patinet et al. (2011) have extended these works by accounting for the effect of the finite dimensions of the specimens in such experiments, using Legrand et al. (2011)'s extension of Rice (1985)'s formula to perturbation of a tensile crack lying on the mid-plane of a plate.

One drawback of the application of the theoretical formulae mentioned above to the interpretation of various experiments was that all of these formulae were accurate only to first order in the perturbation of the front, whereas there was ample evidence that geometric nonlinearities played an important role in most of the experiments. Clearly, such interpretations of experiments would greatly benefit from use of more accurate, higher-order formulae.

In spite of this, a single work may be found in the literature about the second-order coplanar perturbation of a crack, namely that of Adda-Bedia et al. (2006), later summarized by Katzav et al. (2007), devoted to the case of a semi-infinite tensile crack. Unfortunately these papers contained several incompatible second-order formulae. It therefore seems necessary to re-examine the problem.

This paper is devoted to such a re-examination. The method of solution is based on direct and basically straightforward use of earlier results of Rice (1989), and is thus simpler in principle than that of Adda-Bedia et al. (2006), which involved calculation of the second derivatives of the Neuber-Papkovich potentials of the problem with respect to the amplitude of the perturbation. It is therefore believed to be more reliable, and this will be confirmed by finite element computations supporting the new formula that will be found, rather than the old ones.

The paper is organized as follows:

- Section 2 presents the results of Rice (1989) which underlie the treatment. These results consist of two formulae providing, for an arbitrary planar tensile crack, the infinitesimal 
variations of the mode I SIF and fundamental kernel (FK, to be defined below) arising from some infinitesimal coplanar perturbation of the front.

- Section 3 then applies Rice (1989)'s second formula to the calculation of the first-order expression of the FK for a semi-infinite crack with a slightly perturbed front.

- Section 4 next presents the calculation of the second-order expression of the SIF for the same cracked geometry. The principle consists in deducing from Rice (1989)'s first formula, used with the first-order expression of the SIF and FK, the first-order expression of the derivative of the SIF with respect to the amplitude of the perturbation, and then integrating.

- A Fourier transform of the formula found in Section 4 in the direction of the crack front, suggested by the natural translatory invariance of the problem in this direction, is then performed in Section 5 .

- The result of Section 5 is next specialized in Section 6 to the case of a sinusoidal perturbation. The expression found is compared, together with the earlier ones of AddaBedia et al. (2006) and Katzav et al. (2007), to the results of some finite element computations.

- As an application, we finally consider in Section 7 a semi-infinite tensile crack with a periodically but otherwise arbitrarily perturbed front. It is shown that the mean value of the energy-release-rate along this front is exactly the same, up to second order in the perturbation, as if it were straight.

\section{Rice's formulae}

Consider a planar crack with arbitrary contour embedded in some isotropic elastic body $\Omega$ symmetric about the crack plane (Figure 1). Load the crack through some symmetric system of forces and/or displacements prescribed on $\Omega$ and/or $\partial \Omega$. The crack is then in a situation of pure mode I at each point of its front; let $K^{0}(s), s$ being some curvilinear abscissa along this front, denote the local SIF.

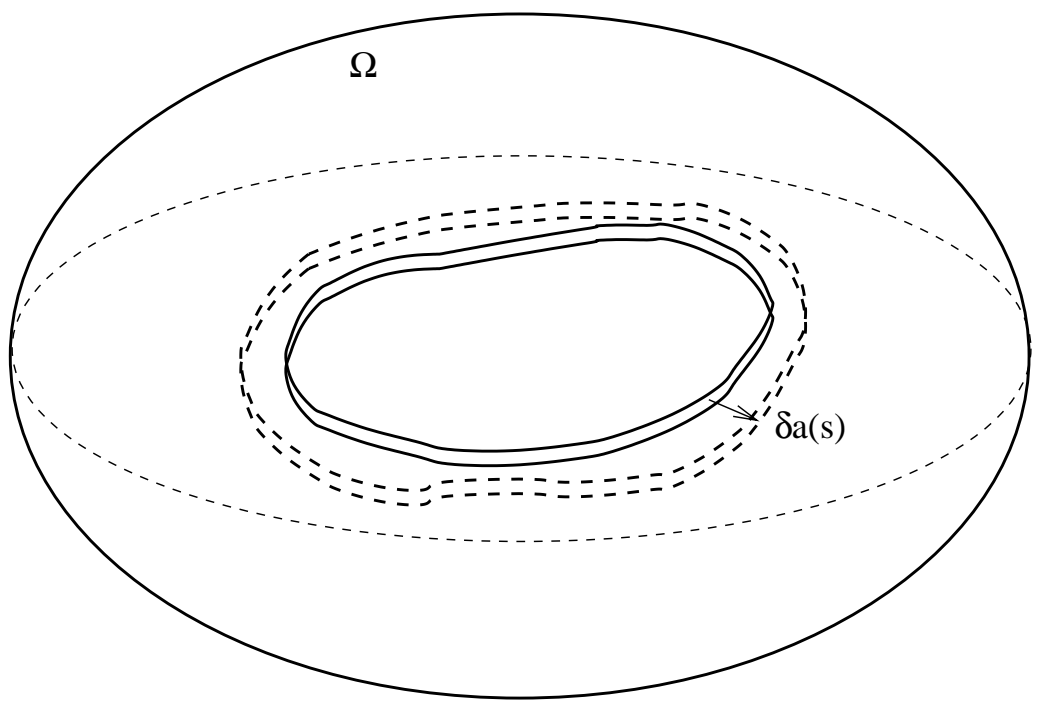

Fig. 1. An arbitrary planar crack with a slightly perturbed front in an arbitrary body 
Now let the front undergo some infinitesimal coplanar perturbation, the loading remaining fixed; let $\delta a(s)$ denote the local orthogonal distance from the original front to the perturbed one (Figure 1). Rice (1989) has shown that under such conditions, the infinitesimal variation $\delta K$ of the local SIF is given by the following equation, referred to as Rice's first formula in the sequel:

$$
\delta K\left(s_{1}\right)=\left[\delta K\left(s_{1}\right)\right]_{\delta a(s) \equiv \delta a\left(s_{1}\right), \forall s}+P V \int_{C F} Z\left(s_{1}, s\right) K^{0}(s)\left[\delta a(s)-\delta a\left(s_{1}\right)\right] d s .
$$

In this expression,

- the integral is taken over the crack front $C F$ and the symbol $P V \int_{C F}$ represents the Cauchy principal value of this integral;

- $\left[\delta K\left(s_{1}\right)\right]_{\delta a(s) \equiv \delta a\left(s_{1}\right), \forall s}$ denotes the value of $\delta K\left(s_{1}\right)$ for a uniform advance of the front equal to $\delta a\left(s_{1}\right)\left(\delta a(s) \equiv \delta a\left(s_{1}\right), \forall s\right)$;

- $Z\left(s_{1}, s\right)$ denotes the fundamental kernel (FK), tied to Bueckner's mode I crack-face weight function.

The FK depends on the cracked geometry considered, but has no dependence upon the loading other than on which portions of $\Omega$ and $\partial \Omega$ have forces versus displacements imposed. It satisfies the following general properties:

$$
Z\left(s_{1}, s_{2}\right)=Z\left(s_{2}, s_{1}\right) \quad ; \quad Z\left(s_{1}, s_{2}\right) \sim \frac{1}{2 \pi\left(s_{1}-s_{2}\right)^{2}} \quad \text { for } s_{1}-s_{2} \rightarrow 0
$$

The second of these properties shows that the integral in equation (1) does make sense as a Cauchy principal value.

In addition, Rice (1989) has shown that provided that the crack advance $\delta a(s)$ vanishes at points $s_{1}$ and $s_{2}$, the infinitesimal variation of the FK is given by the following equation, referred to as Rice's second formula in the sequel:

$$
\delta Z\left(s_{1}, s_{2}\right)=P V \int_{C F} Z\left(s_{1}, s\right) Z\left(s, s_{2}\right) \delta a(s) d s
$$

Note that there are in fact two principal values here, at $s=s_{1}$ and $s=s_{2}$.

Rice's formulae have been used by Bower and Ortiz (1990) and Lazarus (2003) to numerically calculate the SIF along the front of planar tensile cracks with arbitrary contours. The method consisted in starting from some "reference" configuration of this front for which the SIF and FK were known, and then gradually deforming it, updating the SIF and FK at each step, until the configuration of interest was reached. The use made here of Rice's formulae is a kind of analytical equivalent of this numerical method, involving two steps of deformation only. 


\section{$3 \quad$ First-order variation of the fundamental kernel}

\subsection{Generalities}

We now consider the specific case of a semi-infinite tensile crack embedded in some infinite body (Figure 2). The loading consists only of forces imposed at various locations in the body and/or on the crack faces (no prescribed displacements). The initially straight crack front is displaced within the crack plane $O x z$ by a distance $\Delta a(z)$ of the form

$$
\Delta a(z) \equiv A \phi(z)
$$

$A$ being a small, but not necessarily infinitesimal "amplitude" and $\phi(z)$ a given smooth function.

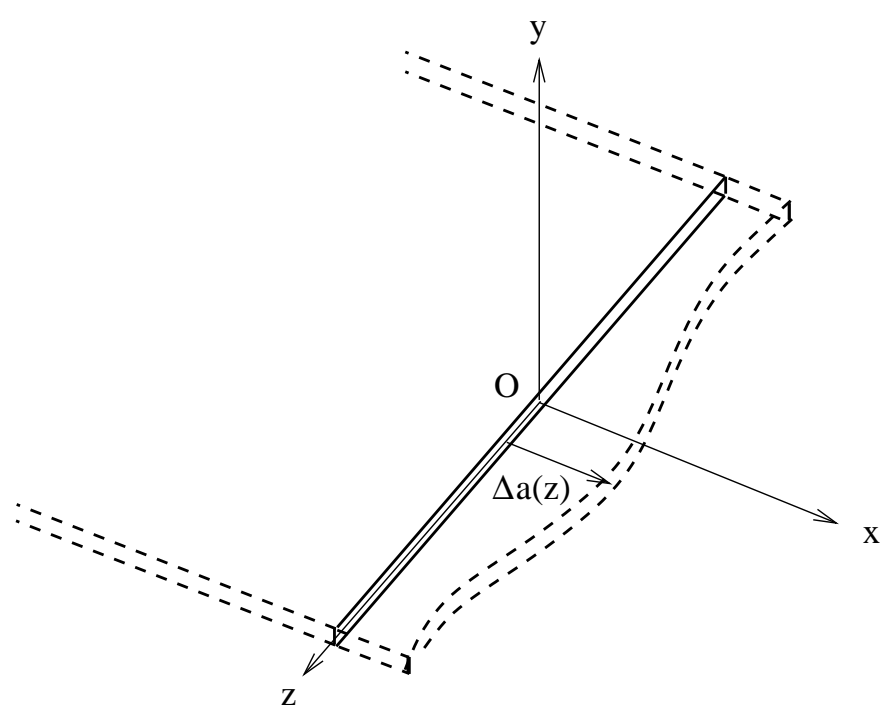

Fig. 2. A semi-infinite crack with a slightly perturbed front in an infinite body

The $\mathrm{FK}$ for this crack configuration is denoted $Z\left(A ; z_{1}, z_{2}\right)$, and our interest here lies in the first-order expression of $Z\left(A ; z_{1}, z_{2}\right)$,

$$
Z\left(A ; z_{1}, z_{2}\right) \equiv Z^{0}\left(z_{1}, z_{2}\right)+A Z^{1}\left(z_{1}, z_{2}\right)+O\left(A^{2}\right)
$$

More precisely, the expression of the $\mathrm{FK} Z^{0}\left(z_{1}, z_{2}\right)$ for the unperturbed configuration of the crack is known to be (Rice, 1985, 1989)

$$
Z^{0}\left(z_{1}, z_{2}\right) \equiv \frac{1}{2 \pi\left(z_{1}-z_{2}\right)^{2}}
$$

and we are looking for that of $Z^{1}\left(z_{1}, z_{2}\right)$. 


\subsection{Application of Rice's second formula}

To be applicable, Rice's second formula (3) requires that the perturbation of the front be zero at those points $z_{1}, z_{2}$ where the variation of the FK is to be evaluated; thus it does not directly apply to the arbitrary perturbation (4). To make up for this difficulty, it suffices, following Rice (1989)'s suggestion, to additively decompose the perturbation $\phi(z)$ into two sub-perturbations:

- one, $\phi_{*}(z)$, consisting of a suitable combination of a translatory motion and a rotation bringing points $z_{1}$ and $z_{2}$ to their correct final positions, given by the two equivalent formulae

$$
\phi_{*}(z) \equiv\left\{\begin{array}{l}
\phi\left(z_{1}\right)+\frac{\phi\left(z_{2}\right)-\phi\left(z_{1}\right)}{z_{2}-z_{1}}\left(z-z_{1}\right) \\
\phi\left(z_{2}\right)+\frac{\phi\left(z_{1}\right)-\phi\left(z_{2}\right)}{z_{1}-z_{2}}\left(z-z_{2}\right) ;
\end{array}\right.
$$

- the other, $\phi(z)-\phi_{*}(z)$, bringing all points to their correct final positions while leaving points $z_{1}$ and $z_{2}$ fixed.

Since our interest lies only in the first-order expression of the FK, the variation of this FK may be obtained by simply adding the variations arising from the two sub-perturbations. Now in the first sub-perturbation, the shape of the front is unchanged, so that the FK remains identical, by equation (6), to the inverse of $2 \pi$ times the squared distance between the points considered; but the variation of this distance is zero to first order in $A$, so that the variation of the FK is zero. On the other hand the second sub-perturbation induces a variation of the FK given by Rice's second formula applied to the perturbation $\phi(z)-\phi_{*}(z)$ instead of $\phi(z)$. It follows that

$$
Z^{1}\left(z_{1}, z_{2}\right)=\frac{1}{4 \pi^{2}} P V \int_{-\infty}^{+\infty} \frac{\phi(z)-\phi_{*}(z)}{\left(z-z_{1}\right)^{2}\left(z-z_{2}\right)^{2}} d z
$$

where use has been made of equation (6).

\subsection{Transformation of the expression of $Z^{1}$}

Although equation (8) may directly be used to evaluate $Z^{1}\left(z_{1}, z_{2}\right)$ numerically, the presence of the rational function $1 /\left[\left(z-z_{1}\right)^{2}\left(z-z_{2}\right)^{2}\right]$ in the integrand makes it unfit for any analytical calculation, whatever the form chosen for the function $\phi(z)$. To get a more convenient form, one must decompose this rational function into partial fractions:

$$
\frac{1}{\left(z-z_{1}\right)^{2}\left(z-z_{2}\right)^{2}}=\frac{1}{\left(z_{1}-z_{2}\right)^{2}}\left[\frac{1}{\left(z-z_{1}\right)^{2}}+\frac{1}{\left(z-z_{2}\right)^{2}}\right]+\frac{2}{\left(z_{1}-z_{2}\right)^{3}}\left(\frac{1}{z-z_{2}}-\frac{1}{z-z_{1}}\right)
$$

and then calculate the integrals of $\phi(z)-\phi_{*}(z)$ times the various fractions, using equation (7) for $\phi_{*}(z)$. When doing so, one must beware that each of these integrals individually diverges at infinity, so that it is momentarily necessary to integrate between large but finite bounds $-L_{1}, L_{2}$ before taking the limit $L_{1}, L_{2} \rightarrow+\infty$ in the final combination of 
integrals. ${ }^{1}$ One thus gets for the fractions $1 /\left(z-z_{1}\right)$ and $1 /\left(z-z_{1}\right)^{2}$, using expression $(7)_{1}$ of $\phi_{*}(z)$ :

$$
\left\{\begin{aligned}
\int_{-L_{1}}^{L_{2}} \frac{\phi(z)-\phi_{*}(z)}{z-z_{1}} d z= & P V \int_{-L_{1}}^{L_{2}} \frac{\phi(z)}{z-z_{1}} d z-\frac{\phi\left(z_{2}\right)-\phi\left(z_{1}\right)}{z_{2}-z_{1}}\left(L_{1}+L_{2}\right) \\
& -\phi\left(z_{1}\right) \ln \frac{L_{2}-z_{1}}{L_{1}+z_{1}} \\
P V \int_{-L_{1}}^{L_{2}} \frac{\phi(z)-\phi_{*}(z)}{\left(z-z_{1}\right)^{2}} d z= & P V \int_{-L_{1}}^{L_{2}} \frac{\phi(z)-\phi\left(z_{1}\right)}{\left(z-z_{1}\right)^{2}} d z-\frac{\phi\left(z_{2}\right)-\phi\left(z_{1}\right)}{z_{2}-z_{1}} \ln \frac{L_{2}-z_{1}}{L_{1}+z_{1}}
\end{aligned}\right.
$$

plus, using equation $(7)_{2}$, similar expressions for the fractions $1 /\left(z-z_{2}\right)$ and $1 /\left(z-z_{2}\right)^{2}$. Using then equation (9) and noting that the terms proportional to $L_{1}+L_{2}$ in the integrals of $\left[\phi(z)-\phi_{*}(z)\right] /\left(z-z_{1}\right)$ and $\left[\phi(z)-\phi_{*}(z)\right] /\left(z-z_{2}\right)$ cancel out, one gets

$$
\begin{aligned}
P V \int_{-L_{1}}^{L_{2}} & \frac{\phi(z)-\phi_{*}(z)}{\left(z-z_{1}\right)^{2}\left(z-z_{2}\right)^{2}} d z= \\
& \frac{1}{\left(z_{1}-z_{2}\right)^{2}}\left[P V \int_{-L_{1}}^{L_{2}} \frac{\phi(z)-\phi\left(z_{1}\right)}{\left(z-z_{1}\right)^{2}} d z+P V \int_{-L_{1}}^{L_{2}} \frac{\phi(z)-\phi\left(z_{2}\right)}{\left(z-z_{2}\right)^{2}} d z\right] \\
& +\frac{2}{\left(z_{1}-z_{2}\right)^{3}}\left[P V \int_{-L_{1}}^{L_{2}} \frac{\phi(z)}{z-z_{2}} d z-P V \int_{-L_{1}}^{L_{2}} \frac{\phi(z)}{z-z_{1}} d z\right] \\
& -\frac{1}{\left(z_{1}-z_{2}\right)^{2}}\left(\frac{\phi\left(z_{2}\right)-\phi\left(z_{1}\right)}{z_{2}-z_{1}} \ln \frac{L_{2}-z_{1}}{L_{1}+z_{1}}+\frac{\phi\left(z_{1}\right)-\phi\left(z_{2}\right)}{z_{1}-z_{2}} \ln \frac{L_{2}-z_{2}}{L_{1}+z_{2}}\right) \\
& +\frac{2}{\left(z_{1}-z_{2}\right)^{3}}\left(\phi\left(z_{1}\right) \ln \frac{L_{2}-z_{1}}{L_{1}+z_{1}}-\phi\left(z_{2}\right) \ln \frac{L_{2}-z_{2}}{L_{1}+z_{2}}\right) .
\end{aligned}
$$

It is easy to check that the sum of the logarithmic terms here goes to zero in the limit $L_{1}, L_{2} \rightarrow+\infty$. One thus gets in this limit, by equation (8):

$$
\begin{aligned}
& Z^{1}\left(z_{1}, z_{2}\right)= \frac{1}{4 \pi^{2}\left(z_{1}-z_{2}\right)^{2}} P V \int_{-\infty}^{+\infty}\left[\frac{\phi(z)-\phi\left(z_{1}\right)}{\left(z-z_{1}\right)^{2}}+\frac{\phi(z)-\phi\left(z_{2}\right)}{\left(z-z_{2}\right)^{2}}\right. \\
&\left.\quad+\frac{2}{z_{1}-z_{2}}\left(\frac{\phi(z)}{z-z_{2}}-\frac{\phi(z)}{z-z_{1}}\right)\right] d z \\
&=\frac{1}{4 \pi^{2}\left(z_{1}-z_{2}\right)^{2}} P V \int_{-\infty}^{+\infty}\left[\left(\frac{1}{z-z_{1}}+\frac{1}{z-z_{2}}\right) \phi^{\prime}(z)\right. \\
&\left.\quad+\frac{2}{z_{1}-z_{2}}\left(\frac{1}{z-z_{2}}-\frac{1}{z-z_{1}}\right) \phi(z)\right] d z
\end{aligned}
$$

where the last expression follows from integration by parts of the first two terms in the preceding integral.

\section{Second-order variation of the stress intensity factor}

We now study the SIF $K\left(A ; z_{1}\right)$ for a semi-infinite tensile crack with a front perturbed in the same way as before, equation (4). Unlike the FK, this SIF depends on the loading imposed. For simplicity, this loading will be assumed to be such that the distribution of

$\overline{1}$ The limit will be perfectly defined since the integral in equation (8) is convergent at infinity, its integrand behaving like $|z|^{-3}$ by equation (7). 
the SIF along the front be invariant when it is displaced by a uniform distance in the direction $x$, be it straight or not; the first, non-integral term in Rice's first formula (1) is then zero, whatever the initial configuration of the front considered. Also, the unperturbed SIF will be assumed to be uniform along the front.

The topic of interest here is the second-order expression of $K\left(A ; z_{1}\right)$,

$$
K\left(A ; z_{1}\right) \equiv K^{0}+A K^{1}\left(z_{1}\right)+A^{2} K^{2}\left(z_{1}\right)+O\left(A^{3}\right) .
$$

More specifically, $K^{0}$ is assumed to be known, the expression of $K^{1}\left(z_{1}\right)$ directly results from Rice's first formula:

$$
K^{1}\left(z_{1}\right)=\frac{K^{0}}{2 \pi} P V \int_{-\infty}^{+\infty} \frac{\phi(z)-\phi\left(z_{1}\right)}{\left(z-z_{1}\right)^{2}} d z=\frac{K^{0}}{2 \pi} P V \int_{-\infty}^{+\infty} \frac{\phi^{\prime}(z)}{z-z_{1}} d z
$$

and we are looking for that of $K^{2}\left(z_{1}\right)$.

To determine the expression of $K^{2}\left(z_{1}\right)$, consider a primary perturbation of the front of the form (4), upon which is added a secondary perturbation

$$
\delta a(z) \equiv \delta A \phi(z)
$$

having the same function $\phi(z)$ as the primary one, but some now infinitesimal amplitude $\delta A$. Then Rice's first formula, applied to the variation of the SIF resulting from the additional perturbation, provides, upon division by $\delta A$, the value of the derivative $(\partial K / \partial A)\left(A ; z_{1}\right)$. The formula involves the SIF and FK for the primarily perturbed configuration of the front. If expressions of these quantities accurate to first order in $A$ are used, the result is the first-order expression of $(\partial K / \partial A)\left(A ; z_{1}\right){ }^{2}$

One thus gets

$$
\begin{aligned}
\frac{\partial K}{\partial A}\left(A ; z_{1}\right)= & P V \int_{-\infty}^{+\infty}\left[Z^{0}\left(z_{1}, z\right)+A Z^{1}\left(z_{1}, z\right)\right]\left[K^{0}+A K^{1}(z)\right]\left[\phi(z)-\phi\left(z_{1}\right)\right] d z+O\left(A^{2}\right) \\
= & K^{0} P V \int_{-\infty}^{+\infty} Z^{0}\left(z_{1}, z\right)\left[\phi(z)-\phi\left(z_{1}\right)\right] d z \\
& +A P V \int_{-\infty}^{+\infty}\left[Z^{0}\left(z_{1}, z\right) K^{1}(z)+Z^{1}\left(z_{1}, z\right) K^{0}\right]\left[\phi(z)-\phi\left(z_{1}\right)\right] d z+O\left(A^{2}\right) .
\end{aligned}
$$

Integration with respect to $A$ then yields

$$
\begin{aligned}
K\left(A ; z_{1}\right)= & K^{0}+A K^{0} P V \int_{-\infty}^{+\infty} Z^{0}\left(z_{1}, z\right)\left[\phi(z)-\phi\left(z_{1}\right)\right] d z \\
& +\frac{A^{2}}{2} P V \int_{-\infty}^{+\infty}\left[Z^{0}\left(z_{1}, z\right) K^{1}(z)+Z^{1}\left(z_{1}, z\right) K^{0}\right]\left[\phi(z)-\phi\left(z_{1}\right)\right] d z+O\left(A^{3}\right),
\end{aligned}
$$

so that

$$
K^{2}\left(z_{1}\right)=\frac{1}{2} P V \int_{-\infty}^{+\infty}\left[Z^{0}\left(z_{1}, z\right) K^{1}(z)+Z^{1}\left(z_{1}, z\right) K^{0}\right]\left[\phi(z)-\phi\left(z_{1}\right)\right] d z .
$$

$\overline{2}$ Note that Rice's formula may safely be applied using the infinitesimal distance between the fronts and the infinitesimal length element measured perpendicularly to, and along the $O z$ axis, respectively, instead of the true ones measured perpendicularly to, and along the primarily perturbed front; indeed the errors made are of second order in $A$. 
The final expression of $K^{2}\left(z_{1}\right)$ is then obtained by using the expressions (6), (10) and (12) of $Z^{0}, Z^{1}$ and $K^{1}$ :

$$
\begin{aligned}
K^{2}\left(z_{1}\right)=\frac{K^{0}}{8 \pi^{2}} P V & \int_{-\infty}^{+\infty} \int_{-\infty}^{+\infty}\left[\left(\frac{1}{z^{\prime}-z_{1}}+\frac{2}{z^{\prime}-z}\right) \phi^{\prime}\left(z^{\prime}\right)\right. \\
& \left.+\frac{2}{z-z_{1}}\left(\frac{1}{z^{\prime}-z_{1}}-\frac{1}{z^{\prime}-z}\right) \phi\left(z^{\prime}\right)\right] \frac{\phi(z)-\phi\left(z_{1}\right)}{\left(z-z_{1}\right)^{2}} d z d z^{\prime}
\end{aligned}
$$

This expression differs from, and is more complex than, formulae (34) and (35) of AddaBedia et al. (2006) (which are themselves distinct).

\section{Formulae in Fourier's space}

The potential usefulness of a formula for the Fourier transform of the second-order variation $K^{2}\left(z_{1}\right)$ in the direction of the crack front is strongly suggested by the natural invariance of the problem in this direction. Such a formula for the first-order variation $K^{1}\left(z_{1}\right)$ has already been extensively used in the literature and proven very useful.

The definition of the Fourier transform $\widehat{\psi}(k)$ of an arbitrary function $\psi(z)$ adopted here is

$$
\psi(z) \equiv \int_{-\infty}^{+\infty} \widehat{\psi}(k) e^{i k z} d k \quad \Leftrightarrow \quad \widehat{\psi}(k) \equiv \frac{1}{2 \pi} \int_{-\infty}^{+\infty} \psi(z) e^{-i k z} d z
$$

For completeness, we first recall the classical expression of the Fourier transform of the first-order variation (see e.g. Lazarus (2011)):

$$
\widehat{K^{1}}(k)=-K^{0} \frac{|k|}{2} \widehat{\phi}(k) .
$$

This expression is easily established by expressing the function $\phi$ in equation $(12)_{2}$ in terms of its Fourier transform $\widehat{\phi}$, and then calculating the integral

$$
\begin{aligned}
P V \int_{-\infty}^{+\infty} \frac{e^{i k z}}{z-z_{1}} d z & =e^{i k z_{1}} P V \int_{-\infty}^{+\infty} \frac{e^{i k\left(z-z_{1}\right)}}{z-z_{1}} d z=e^{i k z_{1}} P V \int_{-\infty}^{+\infty} \frac{\cos \left(k z^{\prime}\right)+i \sin \left(k z^{\prime}\right)}{z^{\prime}} d z^{\prime} \\
& =i \operatorname{sgn}(k) e^{i k z_{1}} \int_{-\infty}^{+\infty} \frac{\sin u}{u} d u=i \pi \operatorname{sgn}(k) e^{i k z_{1}}
\end{aligned}
$$

where $\operatorname{sgn}(x)$ denotes the sign of $x$.

To get the expression of the Fourier transform of the second-order variation, the simplest method again consists in expressing $\phi(z)$ and $\phi\left(z^{\prime}\right)$ in equation (14) in terms of the Fourier transform $\widehat{\phi}$ :

$$
\begin{aligned}
K^{2}\left(z_{1}\right)=\frac{K^{0}}{8 \pi^{2}} \int_{-\infty}^{+\infty} \int_{-\infty}^{+\infty}\left\{P V \int _ { - \infty } ^ { + \infty } \int _ { - \infty } ^ { + \infty } \left[\left(\frac{1}{z^{\prime}-z_{1}}+\frac{2}{z^{\prime}-z}\right) i k^{\prime} \widehat{\phi}\left(k^{\prime}\right) e^{i k^{\prime} z^{\prime}}\right.\right. \\
\left.\left.\quad+\frac{2}{z-z_{1}}\left(\frac{1}{z^{\prime}-z_{1}}-\frac{1}{z^{\prime}-z}\right) \widehat{\phi}\left(k^{\prime}\right) e^{i k^{\prime} z^{\prime}}\right] \widehat{\phi}(k) \frac{e^{i k z}-e^{i k z_{1}}}{\left(z-z_{1}\right)^{2}} d z d z^{\prime}\right\} d k d k^{\prime} .
\end{aligned}
$$

Performing the integration on $z^{\prime}$, we obtain upon use of equation (17): 


$$
\begin{aligned}
K^{2}\left(z_{1}\right)=\frac{K^{0}}{8 \pi} \int_{-\infty}^{+\infty} \int_{-\infty}^{+\infty} \operatorname{sgn}\left(k^{\prime}\right)\left\{P V \int _ { - \infty } ^ { + \infty } \left[-k^{\prime}\left(e^{i k^{\prime} z_{1}}+2 e^{i k^{\prime} z}\right)\right.\right. \\
\left.\left.\quad+\frac{2 i}{z-z_{1}}\left(e^{i k^{\prime} z_{1}}-e^{i k^{\prime} z}\right)\right] \frac{e^{i k z}-e^{i k z_{1}}}{\left(z-z_{1}\right)^{2}} d z\right\} \widehat{\phi}(k) \widehat{\phi}\left(k^{\prime}\right) d k d k^{\prime}
\end{aligned}
$$

To now integrate on $z$, one must calculate the three integrals

$$
\begin{gathered}
P V \int_{-\infty}^{+\infty} \frac{e^{i k z}-e^{i k z_{1}}}{\left(z-z_{1}\right)^{2}} d z \quad, \quad P V \int_{-\infty}^{+\infty} \frac{e^{i k^{\prime} z}\left(e^{i k z}-e^{i k z_{1}}\right)}{\left(z-z_{1}\right)^{2}} d z \\
P V \int_{-\infty}^{+\infty} \frac{\left(e^{i k^{\prime} z_{1}}-e^{i k^{\prime} z}\right)\left(e^{i k z}-e^{i k z_{1}}\right)}{\left(z-z_{1}\right)^{3}} d z
\end{gathered}
$$

this is easily done through integrations by parts and repeated use of formula (17). The result for $K^{2}\left(z_{1}\right)$ reads

$$
\begin{aligned}
K^{2}\left(z_{1}\right)=\frac{K^{0}}{8} & \int_{-\infty}^{+\infty} \int_{-\infty}^{+\infty}\left[\operatorname{sgn}(k) \operatorname{sgn}\left(k^{\prime}\right) k\left(k+k^{\prime}\right)\right. \\
& \left.+\operatorname{sgn}\left(k^{\prime}\right) \operatorname{sgn}\left(k+k^{\prime}\right)\left(k^{\prime 2}-k^{2}\right)-k^{\prime 2}\right] \widehat{\phi}(k) \widehat{\phi}\left(k^{\prime}\right) e^{i\left(k+k^{\prime}\right) z_{1}} d k d k^{\prime} .
\end{aligned}
$$

A nicer formula may be obtained by grouping the terms $\left(k, k^{\prime}\right)$ and $\left(k^{\prime}, k\right)$ together in the double integral, which is equivalent to "symmetrizing" the term [...] with respect to $k$ and $k^{\prime}$ :

where

$$
K^{2}\left(z_{1}\right)=\frac{K^{0}}{8} \int_{-\infty}^{+\infty} \int_{-\infty}^{+\infty} F\left(k, k^{\prime}\right) \widehat{\phi}(k) \widehat{\phi}\left(k^{\prime}\right) e^{i\left(k+k^{\prime}\right) z_{1}} d k d k^{\prime}
$$

$$
\begin{aligned}
F\left(k, k^{\prime}\right) \equiv & \frac{1}{2}\left\{\operatorname{sgn}(k) \operatorname{sgn}\left(k^{\prime}\right)\left(k+k^{\prime}\right)^{2}\right. \\
& \left.+\left[\operatorname{sgn}(k)-\operatorname{sgn}\left(k^{\prime}\right)\right] \operatorname{sgn}\left(k+k^{\prime}\right)\left(k^{2}-k^{\prime 2}\right)-k^{2}-k^{\prime 2}\right\} ;
\end{aligned}
$$

the values of this function in the various regions of the plane $\left(k, k^{\prime}\right)$ are illustrated in Figure 3. Using the change of variable $k_{1} \equiv k+k^{\prime}$, the preceding expression of $K^{2}\left(z_{1}\right)$ may be rewritten in the form

$$
K^{2}\left(z_{1}\right)=\frac{K^{0}}{8} \int_{-\infty}^{+\infty}\left(\int_{-\infty}^{+\infty} F\left(k, k_{1}-k\right) \widehat{\phi}(k) \widehat{\phi}\left(k_{1}-k\right) d k\right) e^{i k_{1} z_{1}} d k_{1}
$$

which implies, upon comparison with the definition $(15)_{1}$ of the Fourier transform, that

$$
\widehat{K^{2}}\left(k_{1}\right)=\frac{K^{0}}{8} \int_{-\infty}^{+\infty} F\left(k, k_{1}-k\right) \widehat{\phi}(k) \widehat{\phi}\left(k_{1}-k\right) d k .
$$

Again, this expression differs from formulae (36) of Adda-Bedia et al. (2006) and (6) of Katzav et al. (2007) (which are themselves distinct).

\section{Case of a sinusoidal perturbation - Numerical validation}

The formulae found for the second-order variation of the SIF ((14) or equivalently (20)) will now be validated through finite element calculations performed for a sinusoidal perturbation of the front. 


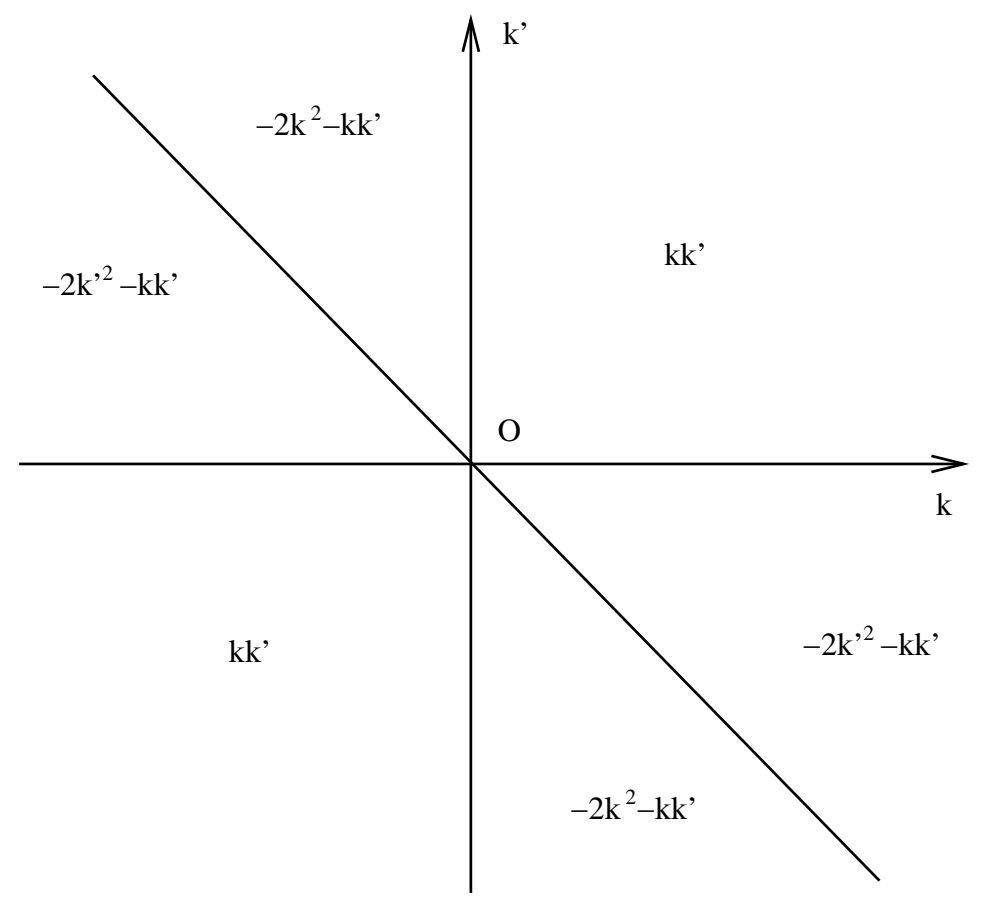

Fig. 3. Values of the function $F\left(k, k^{\prime}\right)$ in the plane $\left(k, k^{\prime}\right)$

The first task is to apply these formulae to such a perturbation. We therefore consider the case where the functions $\phi(z)$ and $\widehat{\phi}(k)$ are of the form

$$
\phi(z) \equiv \cos \left(k_{0} z\right) \quad \Leftrightarrow \quad \widehat{\phi}(k) \equiv \frac{1}{2}\left[\delta\left(k-k_{0}\right)+\delta\left(k+k_{0}\right)\right]
$$

where $k_{0}$ is a positive number and $\delta$ denotes Dirac's function. The first-order variation of the SIF then follows from combination of equations $(15)_{1},(16)$ and $(21)_{2}$ :

$$
\frac{K^{1}\left(z_{1}\right)}{K^{0}}=-\int_{-\infty}^{+\infty} \frac{|k|}{4}\left[\delta\left(k-k_{0}\right)+\delta\left(k+k_{0}\right)\right] e^{i k z_{1}} d k=-\frac{k_{0}}{2} \cos \left(k_{0} z_{1}\right)
$$

and the second-order variation from combination of equations (18) (equivalent to (20)), (19) and $(21)_{2}$ :

$$
\begin{aligned}
\frac{K^{2}\left(z_{1}\right)}{K^{0}}= & \frac{1}{32} \int_{-\infty}^{+\infty} \int_{-\infty}^{+\infty} F\left(k, k^{\prime}\right)\left[\delta\left(k-k_{0}\right)+\delta\left(k+k_{0}\right)\right] \\
& \times\left[\delta\left(k^{\prime}-k_{0}\right)+\delta\left(k^{\prime}+k_{0}\right)\right] e^{i\left(k+k^{\prime}\right) z_{1}} d k d k^{\prime} \\
= & \frac{1}{32}\left[F\left(k_{0}, k_{0}\right) e^{2 i k_{0} z_{1}}+F\left(-k_{0},-k_{0}\right) e^{-2 i k_{0} z_{1}}+F\left(k_{0},-k_{0}\right)+F\left(-k_{0}, k_{0}\right)\right] \\
= & \frac{k_{0}^{2}}{32}\left(e^{2 i k_{0} z_{1}}+e^{-2 i k_{0} z_{1}}-2\right)=-\frac{k_{0}^{2}}{8} \sin ^{2}\left(k_{0} z_{1}\right) .
\end{aligned}
$$

The second-order expansion of the SIF follows from these expressions:

$$
\frac{K\left(A ; z_{1}\right)}{K^{0}}=1-A \frac{k_{0}}{2} \cos \left(k_{0} z_{1}\right)-A^{2} \frac{k_{0}^{2}}{8} \sin ^{2}\left(k_{0} z_{1}\right)+O\left(A^{3}\right) .
$$

The numerical computation will provide the local value of the energy-release-rate $G\left(A ; z_{1}\right)$ rather than that of the SIF. The second-order expansion of $G\left(A ; z_{1}\right)$ is readily deduced 
from that of the square of the SIF, itself resulting from equation (22):

$$
\begin{aligned}
& {\left[\frac{K\left(A ; z_{1}\right)}{K^{0}}\right]^{2}=1-A k_{0} \cos \left(k_{0} z_{1}\right)-A^{2} \frac{k_{0}^{2}}{4} \sin ^{2}\left(k_{0} z_{1}\right)+A^{2} \frac{k_{0}^{2}}{4} \cos ^{2}\left(k_{0} z_{1}\right)+O\left(A^{3}\right)} \\
& =1+A^{2} \frac{k_{0}^{2}}{4}-A k_{0} \cos \left(k_{0} z_{1}\right)-A^{2} \frac{k_{0}^{2}}{2} \sin ^{2}\left(k_{0} z_{1}\right)+O\left(A^{3}\right) \\
& \Rightarrow \quad \frac{G\left(A ; z_{1}\right)}{G^{0}}=A_{0}-A_{1} \cos \left(k_{0} z_{1}\right)-A_{2} \sin ^{2}\left(k_{0} z_{1}\right)+O\left(A^{3}\right) \quad, \quad\left\{\begin{array}{l}
A_{0} \equiv 1+A^{2} \frac{k_{0}^{2}}{4} \\
A_{1} \equiv A k_{0} \\
A_{2} \equiv A^{2} \frac{k_{0}^{2}}{2}
\end{array}\right.
\end{aligned}
$$

where $G^{0}$ denotes the unperturbed energy-release-rate. The comparison with the numerical computations will be performed on the two ratios $A_{1} / A_{0}$ and $A_{2} / A_{1}$, the theoretical values of which are

$$
\frac{A_{1}}{A_{0}}=\frac{A k_{0}}{1+A^{2} k_{0}^{2} / 4} \quad ; \quad \frac{A_{2}}{A_{1}}=\frac{A k_{0}}{2} .
$$

Using the finite element code CAST3M developed by the French Commissariat à l'Energie Atomique (CEA), finite element computations are performed for elastic blocks of the type shown in Figure 4 . The blocks contain an emerging crack the front of which is perturbed according to equation $(21)_{1}$. Because of the periodicity of the problem in the $z$ direction, only one slice of material lying between the planes $z=0$ and $z=\lambda$, where $\lambda \equiv 2 \pi / k_{0}$ denotes the period, is meshed; periodic boundary conditions are imposed on these planes. The other dimensions, that is the depth $a$ of the crack, the length $b$ of the unbroken ligament ahead of the crack front, and the thickness $2 h$ of the block in the vertical direction, are all taken much larger than the period $\lambda$ in order to simulate an infinite body.

Two types of computations are performed with different boundary conditions on the two halves of the left boundary $x=-a$ : opposite bending moments versus opposite vertical displacements. The two types of conditions must yield identical results for large values of the ratios $a / \lambda, b / \lambda$ and $h / \lambda$, and this serves as a test that the values chosen are acceptable.

The two types of computations also use different dimensions, meshes and types of elements. In these involving prescribed moments, the values of the ratios $a / \lambda, b / \lambda$ and $h / \lambda$ are 50, 50 and 10 respectively, and the mesh consists of 96,000 bilinear 6-node prismatic elements and 53,346 nodes. In those involving prescribed displacements, the values $a / \lambda=3,200,{ }^{3} b / \lambda=3,200, h / \lambda=32$ are used, and the mesh consists of 530,100 bilinear 8-node parallelepipedic elements and 598,353 nodes. In both cases the elements are strictly prismatic or parallelepipedic only for the unperturbed straight configuration of the front; for the perturbed configuration the same mesh is used with a slight distortion of elements.

3 Imposing vertical displacements rather than bending moments makes it necessary to use a much larger value of $a / \lambda$, in order to ensure invariance of the SIF in translatory motions of the front in the $x$ direction. 


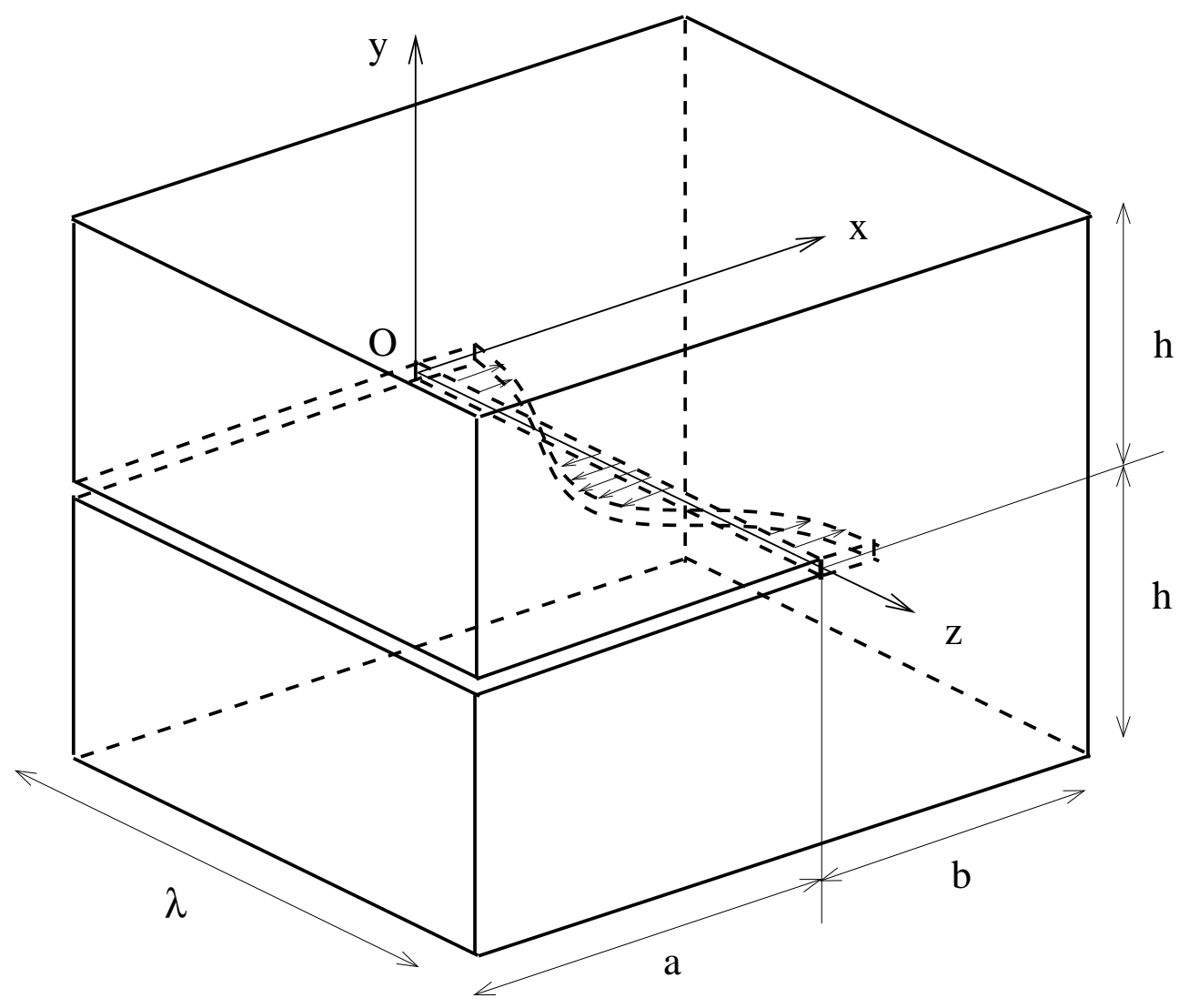

Fig. 4. Geometry considered for the numerical validation

A value of 0.3 is used for Poisson's ratio; the developments above make it clear that the expansion of the SIF is in fact independent of this value. In view of the linearity of the problem, arbitrary values are chosen for the bending moments or vertical displacements imposed.

Local values of the energy-release-rate along the crack front are evaluated using Destuynder et al. (1983)'s $G-\theta$ method, the accuracy of which is well established.

Figure 5 illustrates the results obtained by prescribing bending moments, with a value of the "normalized amplitude of perturbation" $A / \lambda$ of 0.1 . The quantity on the horizontal axis is the normalized distance along the crack front, $z_{1} / \lambda$, and that on the vertical axis is the energy-release-rate $G\left(A ; z_{1}\right)$, expressed in arbitrary units because of the arbitrariness of the magnitude of the loading. The dark blue curve represents numerical results and the red one the sinusoidal approximation of these results having the same maxima and minima. The notable gap between the two is a clear indication that the sinusoidal variation predicted by the first-order expression of $G\left(A ; z_{1}\right) / G_{0}$ represents only a mediocre approximation, and that second-order effects are important.

The numerical values of the ratios $A_{1} / A_{0}$ and $A_{2} / A_{1}$ may easily be deduced from Figure 5. Indeed the sinusoidal approximation of $G\left(A ; z_{1}\right) / G_{0}$ having the same maxima and minima as the true expression (23) of this ratio is simply that obtained by discarding the term $-A_{2} \sin ^{2}\left(k_{0} z_{1}\right)$ in this expression, since this term is precisely zero at the maxima and minima. Hence the red curve simply corresponds to this simplified expression. This means that $A_{0}$ and $A_{1}$ may be identified to the mean value and amplitude of the sinusoidal 


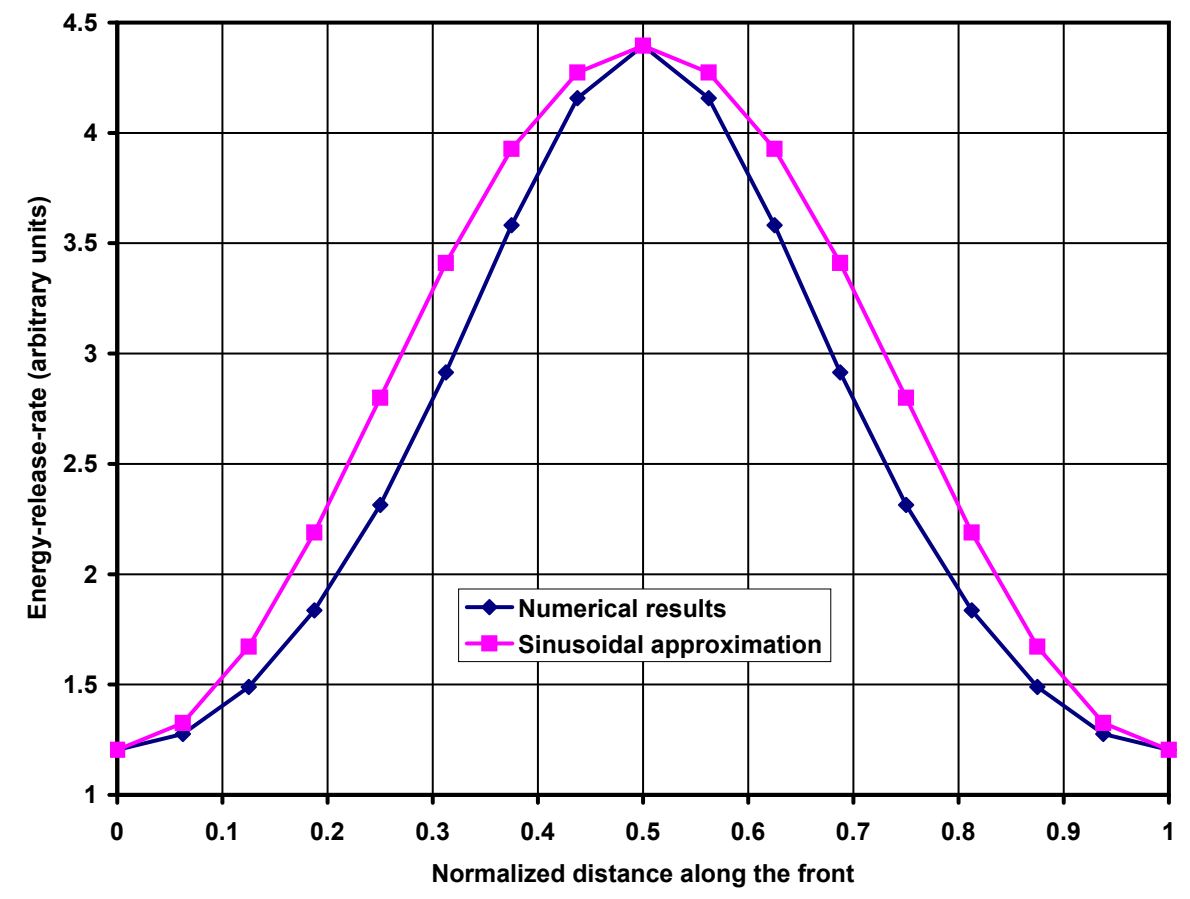

Fig. 5. Distribution of the energy-release-rate along the front - Numerical results and sinusoidal approximation

function represented by the red curve, that is half of the sum and difference of its extremal values. Also, $A_{2}$ may be identified to the maximum vertical gap between the red and dark blue curves.

The numerical values of the ratios $A_{1} / A_{0}$ and $A_{2} / A_{1}$ determined in this way, together with the theoretical values predicted by equations (24), are as follows:

$$
\left\{\begin{array}{l}
\left(A_{1} / A_{0}\right)^{\text {num }}=0.571 \\
\left(A_{1} / A_{0}\right)^{\text {theor }}=0.572
\end{array} ;\left\{\begin{array}{l}
\left(A_{2} / A_{1}\right)^{\text {num }}=0.306 \\
\left(A_{2} / A_{1}\right)^{\text {theor }}=0.314 .
\end{array}\right.\right.
$$

The very good agreement appearing here strongly suggests that all theoretical formulae for the perturbed SIF, (12) and (16) at order 1, (14) and (20) at order 2, are correct. In contrast, formulae (34), (35), (36) of Adda-Bedia et al. (2006) and (6) of Katzav et al. (2007) yield the following theoretical values of the ratio $A_{2} / A_{1}: A k_{0}, A k_{0}, 3 A k_{0} / 2, A k_{0}$, respectively; these values differ from the numerical result $(25)_{3}$ by a factor of 2 or 3 .

As a complement, Figures 6 and 7 show some results obtained by prescribing vertical displacements. The quantities represented are the numerical values of the ratios $A_{1} / A_{0}$ and $A_{2} / A_{1}$, plotted versus the normalized amplitude of perturbation $A / \lambda$ and compared to the theoretical values provided by equations (24). These equations can be observed to provide very good values of $A_{1} / A_{0}$ and $A_{2} / A_{1}$ up to $A / \lambda=0.20$; this means that the second-order formula (23) for $G\left(A ; z_{1}\right) / G_{0}$ gives an accurate representation of this quantity even for normalized amplitudes which are no longer truly small. This is remarkable in view of the fact that for such amplitudes, the variation of $G\left(A ; z_{1}\right) / G_{0}$ along the crack front 
is quite large: see Figure 5 where, even for a lower normalized amplitude $A / \lambda=0.1$, the ratio of the maximum to minimum values of $G\left(A ; z_{1}\right) / G_{0}$ is already of the order of 4 .

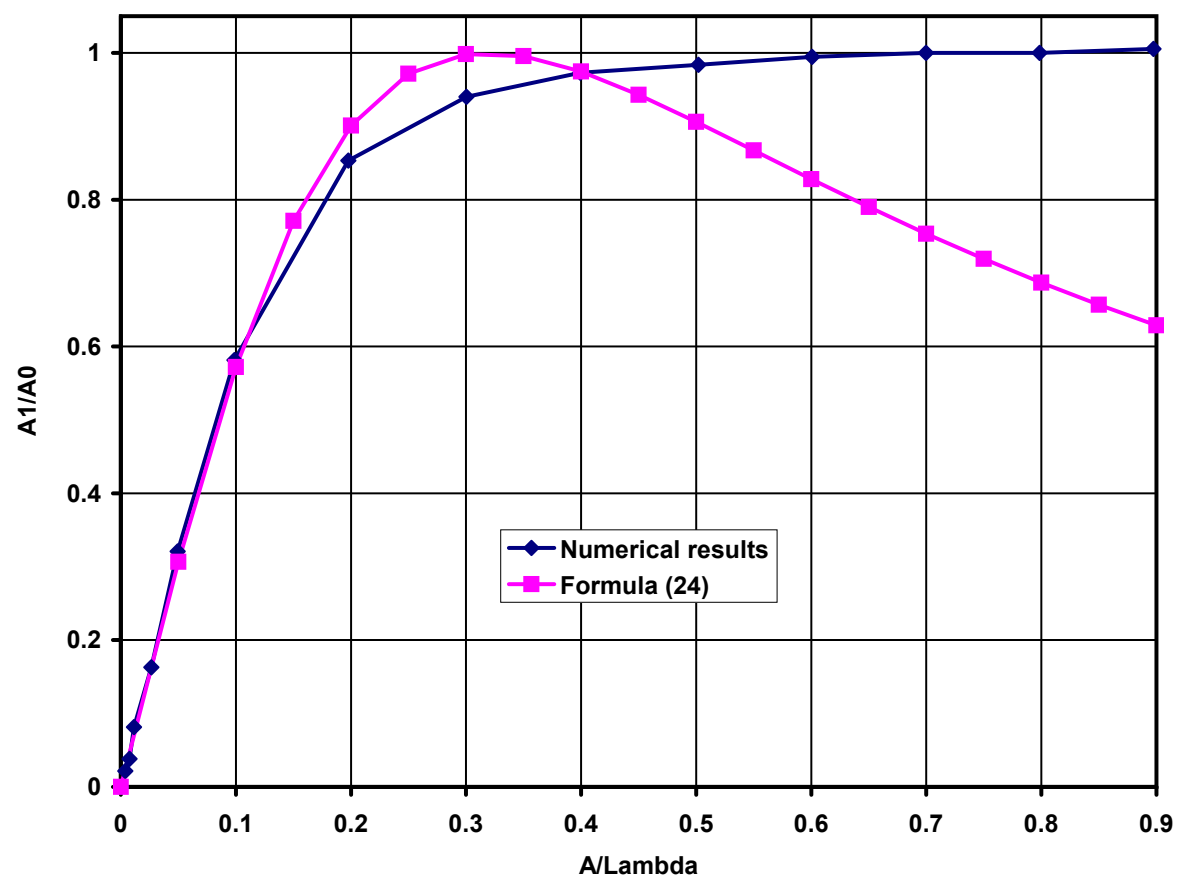

Fig. 6. Ratio $A_{1} / A_{0}$ as a function of the normalized amplitude - Numerical results versus theoretical expression

\section{An application}

As an example of an application, we wish to compare the average values of the energyrelease-rate $G\left(A ; z_{1}\right)$ along the front of a semi-infinite tensile crack in its unperturbed, straight configuration and some arbitrarily perturbed one. More precisely, we shall show that the two mean values are exactly the same up to second order in the perturbation, provided that they are taken with the same infinitesimal weight $d z$. (The other possible choice, for the curved front, is the infinitesimal length element $d s$; the difference between the two quantities is of order $O\left(A^{2}\right)$ and therefore significant).

The definition of the average value of a function defined on the entire real line and varying arbitrarily over it raises difficulties. In order to circumvent them, we shall assume the crack front perturbation to be periodic, of period $2 \pi / k_{0}$ where $k_{0}$ is a positive number; the functions $\phi(z)$ and $\widehat{\phi}(k)$ are then of the form

$$
\phi(z) \equiv \sum_{n=-\infty}^{+\infty} a_{n} e^{i n k_{0} z} \Leftrightarrow \widehat{\phi}(k)=\sum_{n=-\infty}^{+\infty} a_{n} \delta\left(k-n k_{0}\right) .
$$

The energy-release-rate is then also periodic, and its average value over the entire front 


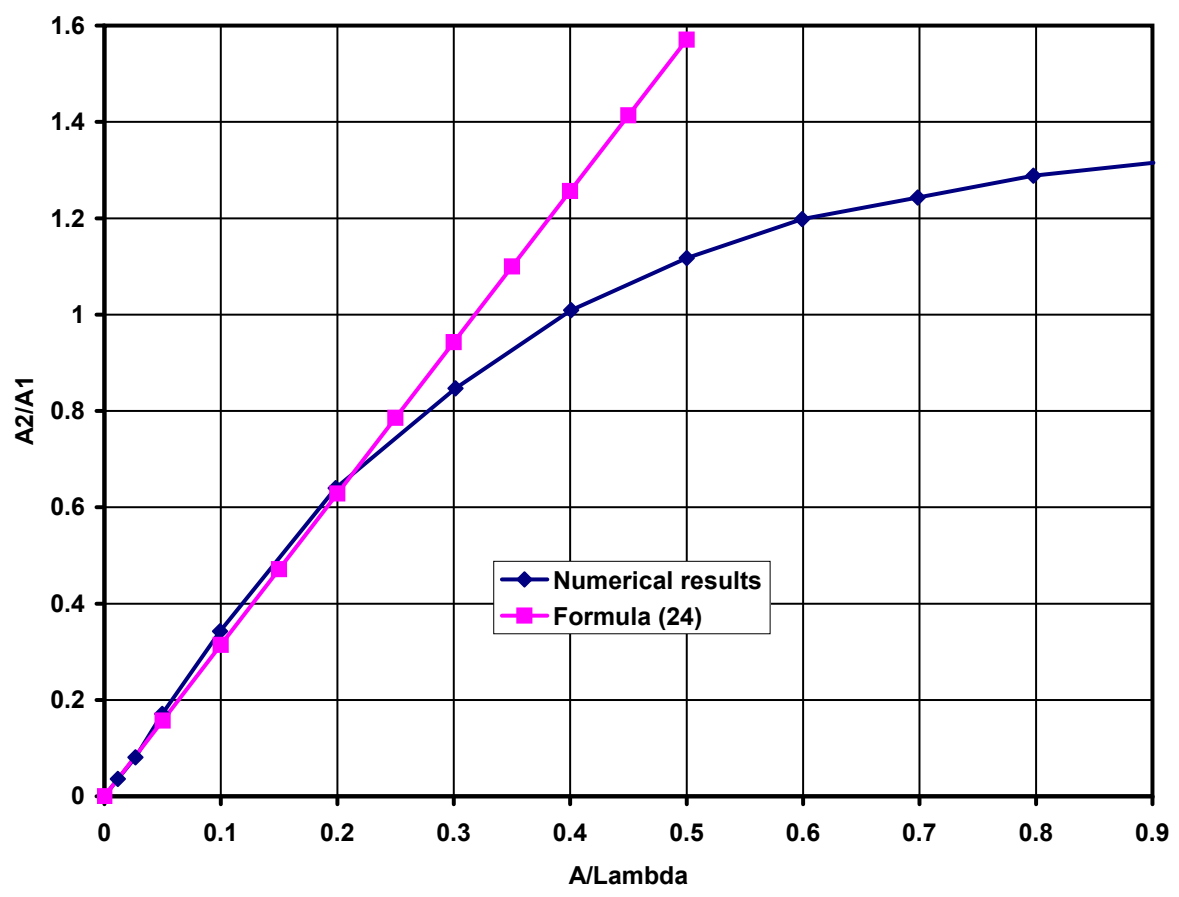

Fig. 7. Ratio $A_{2} / A_{1}$ as a function of the normalized amplitude - Numerical results versus theoretical expression

may be unambiguously defined as its average value over a period.

To derive the expression of this average value, one must use the second-order expansion of the square of the SIF, which reads by equation (11):

$$
\left[\frac{K\left(A ; z_{1}\right)}{K^{0}}\right]^{2}=1+2 A \frac{K^{1}\left(z_{1}\right)}{K^{0}}+2 A^{2} \frac{K^{2}\left(z_{1}\right)}{K^{0}}+A^{2}\left[\frac{K^{1}\left(z_{1}\right)}{K^{0}}\right]^{2}+O\left(A^{3}\right) .
$$

It is thus necessary to evaluate the average values of $K^{1}\left(z_{1}\right) / K^{0},\left[K^{1}\left(z_{1}\right) / K^{0}\right]^{2}$ and $K^{2}\left(z_{1}\right) / K^{0}$.

To calculate the first one, note that by equations $(15)_{1},(16)$ and $(26)_{2}$,

$$
\frac{K^{1}\left(z_{1}\right)}{K^{0}}=-\int_{-\infty}^{+\infty} \frac{|k|}{2} \widehat{\phi}(k) e^{i k z_{1}} d k=-\frac{1}{2} \sum_{n=-\infty}^{+\infty}|n| a_{n} k_{0} e^{i n k_{0} z_{1}}
$$

Now the average value of $e^{i n k_{0} z_{1}}$ is zero or unity depending on whether $n$ differs from, or is equal to zero. Thus in the sum above, only the term $n=0$ could yield a nonzero contribution to the average value, but it does not because of the factor $|n|=0$; therefore

$$
\left\langle\frac{K^{1}\left(z_{1}\right)}{K^{0}}\right\rangle=0
$$

Also, equation (28) implies that 


$$
\left[\frac{K^{1}\left(z_{1}\right)}{K^{0}}\right]^{2}=\frac{1}{4} \sum_{m=-\infty}^{+\infty} \sum_{n=-\infty}^{+\infty}|m n| a_{m} a_{n} k_{0}^{2} e^{i(m+n) k_{0} z_{1}} .
$$

In this double sum, the sole nonzero contributions to the average value arise from the pairs $(m, n)=(-n, n)$. It follows that

$$
\left\langle\left[\frac{K^{1}\left(z_{1}\right)}{K^{0}}\right]^{2}\right\rangle=\frac{1}{4} \sum_{n=-\infty}^{+\infty} n^{2}\left|a_{n}\right|^{2} k_{0}^{2}
$$

where use has been made of the fact that $a_{-n}=\overline{a_{n}}$ since $\phi$ is a real function.

Finally, combination of equations (18) and $(26)_{2}$ yields

$$
\begin{aligned}
\frac{K^{2}\left(z_{1}\right)}{K^{0}}= & \frac{1}{8} \int_{-\infty}^{+\infty} \int_{-\infty}^{+\infty} F\left(k, k^{\prime}\right) \\
& \times\left(\sum_{m=-\infty}^{+\infty} \sum_{n=-\infty}^{+\infty} a_{m} \delta\left(k-m k_{0}\right) a_{n} \delta\left(k^{\prime}-n k_{0}\right)\right) e^{i\left(k+k^{\prime}\right) z_{1}} d k d k^{\prime} \\
= & \frac{1}{8} \sum_{m=-\infty}^{+\infty} \sum_{n=-\infty}^{+\infty} F\left(m k_{0}, n k_{0}\right) a_{m} a_{n} e^{i(m+n) k_{0} z_{1}} .
\end{aligned}
$$

Again, the sole nonzero contributions to the average value arise from the pairs $(m, n)=$ $(-n, n)$ so that

$$
\left\langle\frac{K^{2}\left(z_{1}\right)}{K^{0}}\right\rangle=\frac{1}{8} \sum_{n=-\infty}^{+\infty} F\left(-n k_{0}, n k_{0}\right) a_{-n} a_{n}=-\frac{1}{8} \sum_{n=-\infty}^{+\infty} n^{2}\left|a_{n}\right|^{2} k_{0}^{2}
$$

where equation (19) (or Figure 3) has been used.

Combining equations (27), (29), (30) and (31), one sees that

$$
\left\langle\left[\frac{K\left(A ; z_{1}\right)}{K^{0}}\right]^{2}\right\rangle \equiv\left\langle\frac{G\left(A ; z_{1}\right)}{G^{0}}\right\rangle=1+O\left(A^{3}\right)
$$

where $G^{0}$ denotes the unperturbed energy-release-rate; this establishes the property announced.

The meaning of this property is that when the crack front propagates without any deformation over some pre-specified distance $a$ under constant loading, the total (elastic+potential) energy released is independent of whether this front is straight or (periodically) curved.

This result could be anticipated using a simple argument sketched in a paper of Gao and Rice (1989) and explained in more detail below. Figure 8 shows the trace of the crack plane within a slice of material of thickness equal to the period $\lambda$ of the crack front perturbation, and four possible configurations of the front, two straight ones $S_{1}, S_{2}$ separated by a distance of $a$, and two identically curved ones $C_{1}, C_{2}$ separated by the same distance.

The loading being fixed, let $W_{S}^{1}$ and $W_{S}^{2}$ denote the total (elastic+potential) energies of 


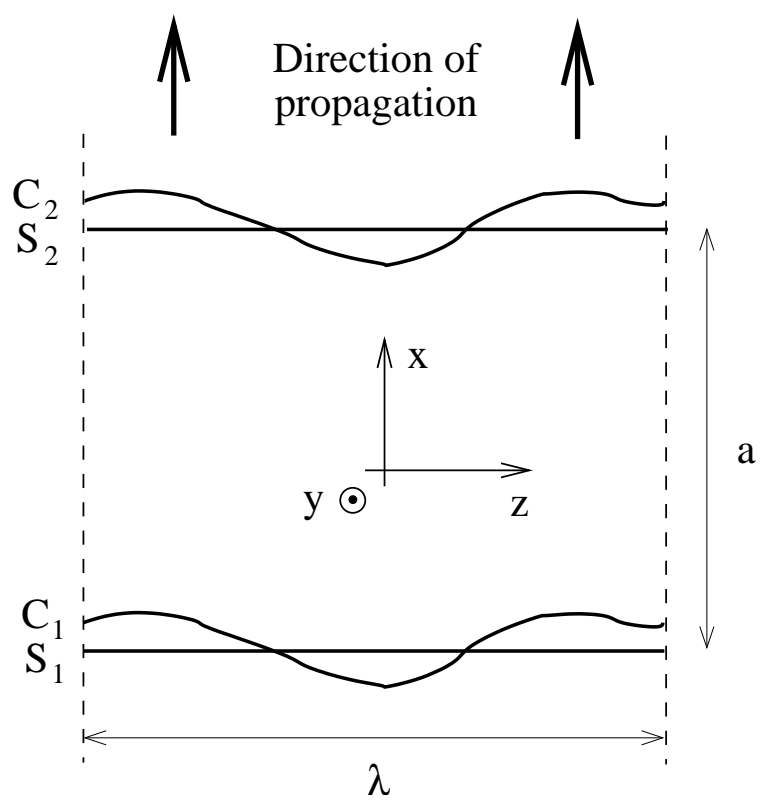

Fig. 8. Translational motions of a straight front and a periodically perturbed one

the slice for the configurations $S_{1}$ and $S_{2}$ of the front, and $W_{C}^{1}$ and $W_{C}^{2}$ those for the configurations $C_{1}$ and $C_{2}$. By definition of the energy-release-rate,

$$
W_{S}^{1}-W_{S}^{2}=G^{0} a \lambda \quad ; \quad W_{C}^{1}-W_{C}^{2}=\left\langle G\left(A ; z_{1}\right)\right\rangle a \lambda
$$

where the average value is taken with the infinitesimal weight $d z$ (not $d s$ ). Taking the difference between these equations, one gets

$$
W_{C}^{1}-W_{S}^{1}-\left(W_{C}^{2}-W_{S}^{2}\right)=\left[\left\langle G\left(A ; z_{1}\right)\right\rangle-G^{0}\right] a \lambda .
$$

But the difference $W_{C}^{1}-W_{S}^{1}$ represents the variation of energy of the slice caused by the deformation of the front from the straight configuration $S_{1}$ to the curved one $C_{1}$; and $W_{C}^{2}-W_{S}^{2}$ admits a similar interpretation. Now the invariance of the problem in the direction $x$ implies that such a difference is unaffected by translatory motions of the front in this direction, so that $W_{C}^{1}-W_{S}^{1}=W_{C}^{2}-W_{S}^{2}$. The preceding equation then implies that

$$
\left\langle G\left(A ; z_{1}\right)\right\rangle=G^{0}
$$

This reasoning makes it clear that the property discussed is related to the invariance of the problem considered in the direction of crack propagation, and would not subsist for other geometries and/or loadings. On the other hand it may be observed to be independent of the magnitude of the perturbation, and thus hold at all orders in $A$.

A final observation is that unlike formulae (14) and (20), none of those of Adda-Bedia et al. (2006) and Katzav et al. (2007) satisfies property (32). This brings additional support to the former formulae since the correctness of the property has been established by independent means. 


\section{Conclusion}

This paper was devoted to some re-derivation of the expression of the second-order variation of the local stress intensity factor (SIF) resulting from coplanar perturbation of the front of a semi-infinite tensile crack in some infinite body. The task seemed necessary in view of the fact that the earlier work of Adda-Bedia et al. (2006) on the topic, as well as Katzav et al. (2007)'s summary of it, contained inconsistencies.

The method of derivation, which consisted in some basically straightforward application of some results of Rice (1989), was simpler in principle than that of Adda-Bedia et al. (2006), which implied calculation of the second derivatives of the Neuber-Papkovich potentials of the problem with respect to the amplitude of the perturbation.

The results of Rice (1989) used were twofold. The first one was a formula for the infinitesimal variation of the local mode I SIF resulting from infinitesimal coplanar perturbation of an arbitrary planar crack, involving the fundamental kernel (FK) of the cracked geometry considered. The second one was a similar formula for the infinitesimal variation of the FK.

In a first step, Rice's second formula was used to derive an expression of the FK for a semi-infinite crack with a slightly curved front, accurate to first order in the deviation from straightness.

In a second step, Rice's first formula was applied to the same perturbed configuration of the front with the first-order expressions of the SIF and FK, to derive the first-order expression of the derivative of the SIF with respect to the amplitude of the perturbation. Integration with respect to this amplitude then yielded the second-order expression of the perturbed SIF.

A Fourier transform of this second-order expression in the direction of the crack front, suggested by the natural invariance of the problem in this direction, was then performed.

The next step consisted in applying the formula for the Fourier transform of the secondorder variation of the local SIF to some sinusoidal perturbation of the front. Finite element computations were then performed for solid blocks of large dimensions, simulating an infinite body, and containing such a sinusoidally perturbed crack. The numerical results were found to bring support to the new formula for the second-order variation of the local SIF, rather than the earlier ones of Adda-Bedia et al. (2006) and Katzav et al. (2007).

As a final application, we considered a semi-infinite crack with a periodically perturbed front, and calculated the average value of the energy-release rate along this front up to second order in the deviation from straightness. This average value was found to exactly coincide with that for a straight front, and a simple rationale for the coincidence was provided. This observation and explanation brought additional support to the new formula for the second-order variation of the local SIF.

\section{Acknowledgement}


The authors wish to express their sincere thanks to Drs. Mokhtar Adda-Bedia, Laurent Ponson and Damien Vandembroucq for several stimulating discussions.

\section{References}

Adda-Bedia M., Katzav E. and Vandembroucq D. (2006). Second-order variation in elastic fields of a tensile planar crack with a curved front. Phys. Rev. E, 73, 035106(1-4).

Alava M.J., Nukala P.K. and Zapperi S. (2006). Statistical models of fracture. Adv. Phys., 55, 349-476.

Bonamy D., Santucci S. and Ponson L. (2008). Cracking dynamics in material failure as the signature of a self-organized dynamic phase transition. Phys. Rev. Lett., 101, 045501.

Bower A.F. and Ortiz M. (1990). Solution of three-dimensional crack problems by a finite perturbation method. J. Mech. Phys. Solids, 38, 443-480.

Charles Y., Vandembroucq D., Hild F. and Roux S. (2004). Material-independent crack arrest statistics. J. Mech. Phys. Solids, 52, 1651-1669.

Destuynder P., Jaoua M. and Lescure S. (1983). Quelques remarques sur la mécanique de la rupture élastique. Journal de Mécanique Théorique et Appliquée, 2, 113-135 (in French).

Favier E., Lazarus V. and Leblond J.B. (2006). Statistics of the deformation of the front of a tunnel-crack propagating in some inhomogeneous medium. J. Mech. Phys. Solids, 54, 1449-1478.

Gao H. (1988). Nearly circular shear mode cracks. Int. J. Solids Structures, 24, 177-193.

Gao H. and Rice J.R. (1986). Shear stress intensity factors for planar crack with slightly curved front. ASME J. Appl. Mech., 53, 774-778.

Gao H. and Rice J.R. (1987a). Somewhat circular tensile cracks. Int. J. Fracture, 33, $155-174$.

Gao H. and Rice J.R. (1987b). Nearly circular connections of elastic half-spaces. ASME J. Appl. Mech., 54, 627-634.

Gao H. and Rice J.R. (1989). A first-order perturbation analysis of crack trapping by arrays of obstacles. ASME J. Appl. Mech., 56, 828-836.

Katzav E. and Adda-Bedia M. (2006). Roughness of tensile crack fronts in heterogenous materials. Europhys. Lett., 76, 450-456.

Katzav E., Adda-Bedia M., Ben Amar M. and Boudaoud A. (2007). Roughness of moving elastic lines: crack and wetting fronts. Phys. Rev. E, 76, 051601(1-12).

Krishnamurthy S., Tanguy A. and Roux S. (2000). Dynamic exponent in extremal models of pinning. Eur. Phys. J. B, 15, 149-153.

Laurson L., Santucci S. and Zapperi S. (2010). Avalanches and clusters in planar crack front propagation. Phys. Rev. E, 81, 046116.

Lazarus V. (2003). Brittle fracture and fatigue propagation paths of 3D plane cracks under uniform remote tensile loading. Int. J. Fracture, 122, 23-46.

Lazarus V. (2011). Perturbation approaches of a planar crack in linear fracture mechanics: a review. J. Mech. Phys. Solids, 59, 121-144.

Lazarus V. and Leblond J.B. (1998a). Three-dimensional crack-face weight functions for the semi-infinite interface crack. I. Variation of the stress intensity factors due to some small perturbation of the crack front. J. Mech. Phys. Solids, 46, 489-511. 
Lazarus V. and Leblond J.B. (1998b). Three-dimensional crack-face weight functions for the semi-infinite interface crack. II. Integro-differential equations on the weight functions and resolution. J. Mech. Phys. Solids, 46, 513-536.

Lazarus V. and Leblond J.B. (2002a). In-plane perturbation of the tunnel-crack under shear loading. I: Bifurcation and stability of the straight configuration of the front. Int. J. Solids Structures, 39, 4421-4436.

Lazarus V. and Leblond J.B. (2002b). In-plane perturbation of the tunnel-crack under shear loading. II: Determination of the fundamental kernel. Int. J. Solids Structures, 39, 4437-4455.

Leblond J.B., Mouchrif S.E. and Perrin G. (1996). The tensile tunnel-crack with a slightly wavy front. Int. J. Solids Structures, 33, 1995-2022.

Legrand L. and Leblond J.B. (2010a). In-plane perturbation of a system of two coplanar slit-cracks - II: Case of close inner crack fronts or distant outer ones. Int. J. Solids Structures, 47, 3504-3512.

Legrand L. and Leblond J.B. (2010b). Evolution of the shape of the fronts of a pair of semi-infinite cracks during their coplanar coalescence. Zeitschrift für Angewandte Mathematik und Mechanik, 90, 821-836.

Legrand L., Patinet S., Leblond J.B., Frelat J., Lazarus V. and Vandembroucq D. (2011). Coplanar perturbation of a crack lying on the mid-plane of a plate. Int. J. Fracture, 170, 67-82.

Patinet S., Frelat J., Lazarus V. and Vandembroucq D. (2011). Propagation des fronts de fissure plane dans les matériaux fragiles hétérogènes de dimensions finies. Mécanique et Industries, 12, 199-204 (in French).

Piccolroaz A., Mishuris G. and Movchan A.B. (2007). Evaluation of the Lazarus-Leblond constants in the asymptotic model of the interfacial wavy crack. J. Mech. Phys. Solids, 55, 1575-1600.

Pindra N., Lazarus V. and Leblond J.B. (2008). The deformation of the front of a 3D interface crack propagating quasistatically in a medium with random fracture properties. J. Mech. Phys. Solids, 56, 1269-1295.

Pindra N., Lazarus V. and Leblond J.B. (2009). Geometrical disorder of the fronts of a tunnel-crack propagating in shear in some heterogeneous medium. J. Mech. Phys. Solids, 58, 281-299.

Pindra N., Lazarus V. and Leblond J.B. (2010). In-plane perturbation of a system of two coplanar slit-cracks - I: Case of arbitrarily spaced crack fronts. Int. J. Solids Structures, 47, 3489-3503.

Ponson L. and Bonamy D. (2010). Crack propagation in brittle heterogeneous solids: material disorder and crack dynamics. Int. J. Fracture, 162, 21-31.

Rice J.R. (1985). First-order variation in elastic fields due to variation in location of a planar crack front. ASME J. Appl. Mech., 52, 571-579.

Rice J.R. (1989). Weight function theory for three-dimensional elastic crack analysis. In: Wei and Gangloff (eds.), Fracture Mechanics: Perspectives and Directions (Twentieth Symposium), American Society for Testing and Materials STP 1020, Philadelphia, pp. 29-57.

Roux S., Vandembroucq D. and Hild F. (2003). Effective toughness of heterogeneous brittle materials. Eur. J. Mech. A/Solids, 22, 743-749.

Schmittbuhl J., Delaplace A., Maloy K.J., Perfettini H. and Vilotte J.P. (2003). Slow crack propagation and slip correlations. Pure Appl. Geophys., 160, 961-976. 
Schmittbuhl J., Roux S., Vilotte J.P. and Maloy K.J. (1995). Interfacial crack pinning: effect of nonlocal interactions. Phys. Rev. Lett., 74, 1787-1790.

Schmittbuhl J. and Vilotte J.P. (1999). Interfacial crack front wandering: influence of quenched noise correlations. Physica A, 270, 42-56.

Tanguy A., Gounelle M. and Roux S. (1998). From individual to collective pinning: effect of long-range elastic interactions. Phys. Rev. E, 58, 1577-1590. 